\title{
Reverse shoulder arthroplasty with os acromiale
}

\author{
Woo-Jin Shin, Hyun-Ju Lee, Ki-Yong An \\ Department of Orthopedic Surgery, Gwangju Veterans Hospital, Gwangju, Korea
}

\begin{abstract}
Function and strength of the deltoid muscle are important in reverse shoulder arthroplasty (RSA). Moreover, location and shape of the acromion, clavicle, and scapular spine, which are origins of the deltoid muscle, are also important. The frequency of os acromiale is 5\% to $15 \%$; however, it is rare in the Asian population, affecting approximately $0.7 \%$ of Koreans. RSA has rarely been reported in patients with os acromiale. We present a case series of two patients with cuff tear and arthropathy combined with os acromiale who underwent RSA. From 2016 to 2018, two patients with os acromiale who presented with pain and limited range of motion (ROM) underwent RSA with cuff tear arthropathy using the subscapularis-sparing deltopectoral approach. Their ROM, visual analog scale (VAS), and satisfaction were evaluated before and after surgery. In both patients, VAS decreased, ROM increased, and postoperative satisfaction increased. There were no specific complications due to os acromiale. The VAS, ROM, and satisfaction of patients improved after surgery compared with values before surgery. However, careful attention must be given during surgery to ensure optimal repair and recovery.
\end{abstract}

Keywords: Os acromiale; Cuff tear arthropathy; Reverse shoulder arthroplasty

Os acromiale is a disease caused by failure of osseous union at the ossification center of the acromion. It is divided into preacromion, meso-acromion, meta-acromion, and basiacromion areas and is classified based on area (Fig. 1) [1,2]. The prevalence of os acromiale varies from $1.9 \%$ to $15 \%$ depending on population [3]. The condition is usually asymptomatic and is incidentally found on radiography, magnetic resonance imaging, or computed tomography, which is typically performed to diagnose shoulder cuff or other shoulder problems [4].

If treatment with reverse shoulder arthroplasty (RSA) is required, such as in cases of irreparable rotator cuff tears or cuff tear arthropathy, the tension in the deltoid muscle may increase, and the os acromiale, the origin of the deltoid muscle, may affect deltoid function and must be considered when planning treat- ment. We performed RSA in two patients two patients with os acromiale, using the previously published subscapularis-sparing deltopectoral approach [5], and compared the parameters before and after RSA.

\section{CASE REPORT}

All procedures were part of the standard medical care, and the need for ethics approval and consent to participate were waived. Written informed consent was obtained from the patients for publication of this case report and any accompanying images.

\section{Case 1}

A 71-year-old man presented with pain and limitation in range of motion (ROM) in his left shoulder, with no history of previous

Received: December 2, 2019 Revised: December 2, 2019 Accepted: February 10, 2020

Correspondence to: Ki-Yong An

Department of Orthopedic Surgery, Gwangju Veterans Hospital, 99 Cheomdanwolbong-ro, Gwangsan-gu, Gwangju 62284, Korea

Tel: +82-62-602-6162, Fax: +82-62-602-6164, E-mail: mdaky@ hanmail.net, ORCID: https://orcid.org/0000-0003-3583-6425

\section{Financial support: None.}

Conflict of interest: None.

Copyright@ 2020 Korean Shoulder and Elbow Society. All Rights Reserved.

This is an Open Access article distributed under the terms of the Creative Commons Attribution Non-Commercial License (http://creativecommons.org/licenses/by-nc/4.0/) which permits unrestricted non-commercial use, distribution, and reproduction in any medium, provided the original work is properly cited. 
trauma or operation. The patient stated that the pain had been persistent for about 7 years, for which he had been undergoing conservative treatment with analgesic medication. Initially, the pain was bearable but gradually increased. Limitation of movement had also increased.

Physical examination of the left shoulder revealed no gross deformity. Neer and Hawkins tests, external rotation lag sign, and pseudoparalysis assessment were positive. Passive ROM was almost normal, and active ROM was $10^{\circ}$ flexion, $30^{\circ}$ abduction, and internal rotation at the buttock level. The functional assessment score was 7 points on the visual analog scale (VAS).

Imaging exams of radiography and magnetic resonance imaging revealed upward migration of the humeral head, glenohu-

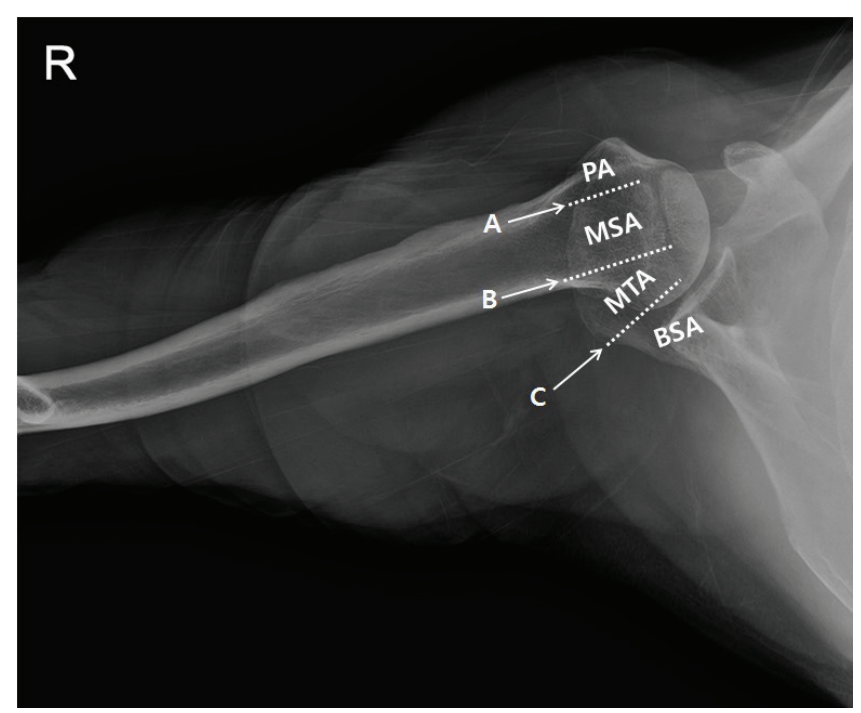

Fig. 1. Os acromion subtypes: pre-acromial (PA), meso-acromial (MSA), meta-acromial (MTA), basi-acromial (BSA). Depending on location of the osseous union at the ossification center of the acromion, the condition is classified as type A, type B, and type C. meral osteoarthritis, acetabulization (acromial thinning), and meso-acromion (Fig. 2). A nonsurgical treatment approach with analgesics and rest was used for several years with no clinical improvement. Thus, surgical treatment was recommended. The surgical technique for RSA is as follows. The patient was placed in the beach-chair position, and RSA was performed using the deltopectoral approach with preservation of the subscapularis tendon.

A straight incision was placed approximately $10 \mathrm{~cm}$ from the lateral edge of the coracoid to the insertion site of the humerus along the anterior border of the deltoid, followed by deep dissection through the deltopectoral groove to the lateral side of the cephalic vein. The conjoint tendon was retracted to the medial side, the Hohmann retractor was placed in the subacromial space, the surrounding soft tissue was removed, and the head of the humerus was exposed.

After resection of the long head of the biceps tendon, the humeral head was pushed up to the rotator interval, the supraspinatus and infraspinatus were pushed apart, and the humeral head was completely exposed. Bone cutting was performed at the anatomical neck level using the resection guide. After cutting the humeral head and pressing the humerus downward, the glenoid was exposed, and glenoid reaming was performed after removing the glenoid articular surface and labrum. The baseplate and hemisphere were inserted, a humoral stem was installed, and stability, conjoint tension, and ROM were measured after joint reduction. The wound was closed after irrigation, and the surgery was completed after closed suction drainage.

Shoulder movement was not restricted after surgery. Immediately after the operation, the arm was placed in an arm sling with no abduction brace, and exercise was started immediately in the range with the least amount of pain. After stitches were removed,

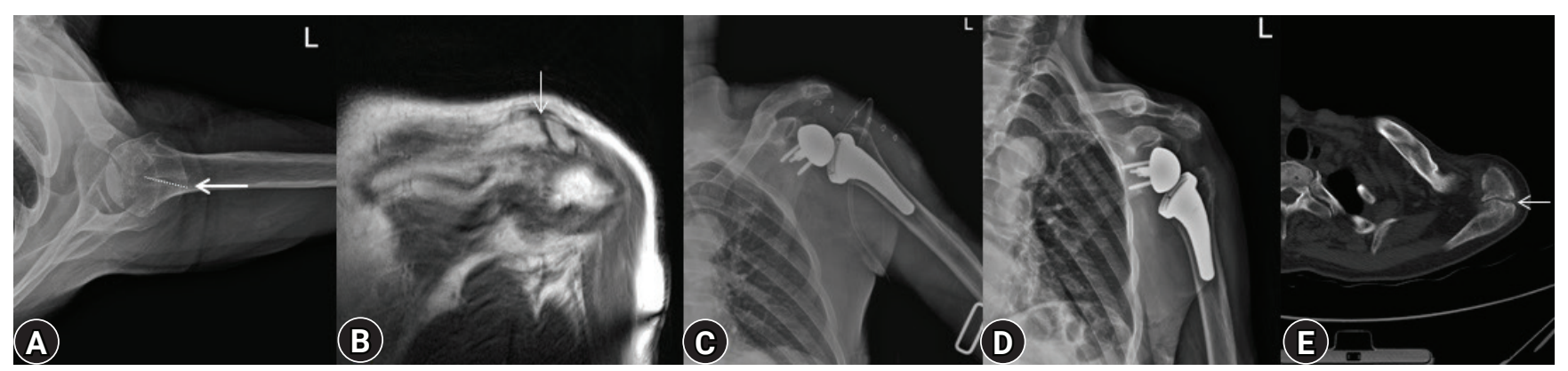

Fig. 2. A 71-year-old man with a left shoulder, full thickness rotator cuff tear underwent reverse shoulder arthroplasty, revealing os acromiale of meso-acromion type. (A) Preoperative axillary view X-ray showed meso-acromion findings. (B) T1-weighted coronal plane image showed a meso-acromion finding. (C) Anteroposterior postoperative X-ray showed no significant difference in the acromion before and after surgery. (D) One year later, the acromiale showed no morphological changes on the anteroposterior postoperative X-ray. (E) One year later, the acromiale showed no morphological changes on the postoperative computed tomography. Undefined (arrows, meso-acromion). 
active ROM exercises were initiated, and no special physical treatments were performed. One year after the surgery, the postoperative parameters were satisfactory, with ROM flexion $160^{\circ}$, abduction $160^{\circ}$, internal rotation at the L5 level, and VAS score of 0 . The University of California at Los Angeles (UCLA) shoulder score increased significantly from preoperative 7 of 35 (20.0\%) to postoperative 31 of 35 (88.6\%).

\section{Case 2}

A 73-year-old man presented with pain and limitation in ROM of his right shoulder. His shoulder problem had started about 20 years earlier, and there was no history of trauma. He had undergone right arthroscopic cuff repair due to complete cuff tear about 8 years prior and open reduction with plate fixation due to right olecranon fracture about 10 years prior to presentation.

Initially, the pain was partially relieved with use of analgesics and steroid injection therapy, but increasing shoulder pain followed. Physical examination revealed positive results of the Neer, Hawkins, empty can, and lift off tests of the right shoulder. Preoperative ROM was flexion $70^{\circ}$, abduction $90^{\circ}$, internal rotation at L5 level, and VAS score of 6 . The os acromion type was B.

$\mathrm{X}$-ray radiography images revealed upward migration of the humeral head, glenohumeral osteoarthritis, acetabulization, and os acromion type B (Fig. 3). Based on his condition, surgical treatment was decided as the best approach. Surgery and postoperative treatment were performed in the same way as discussed for case 1. The postoperative parameters were flexion $140^{\circ}$, abduction $140^{\circ}$, and internal rotation at $\mathrm{L} 1$ at 1 year postoperative. The patient was
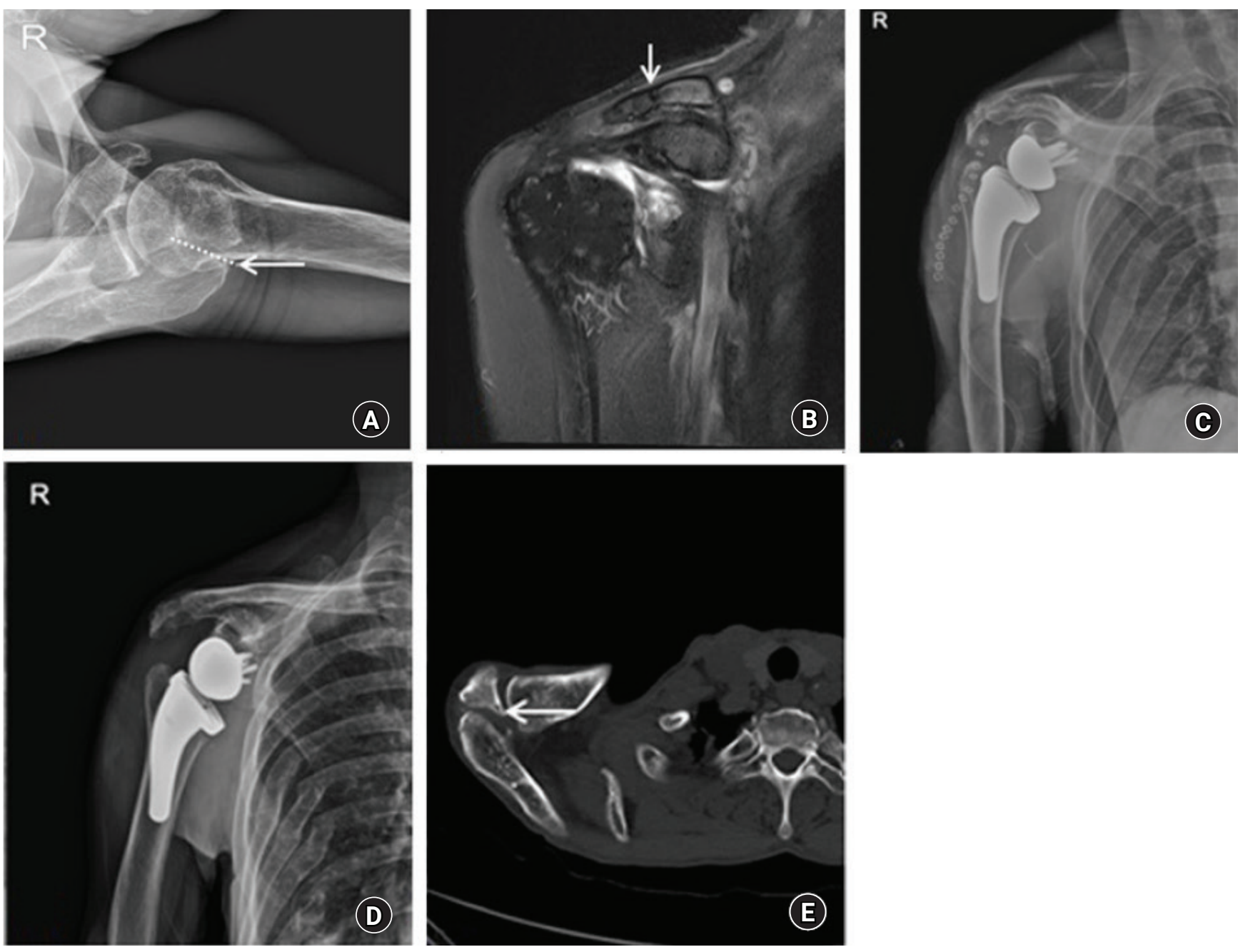

Fig. 3. A 73-year-old man with a right shoulder, full thickness rotator cuff tear underwent reverse shoulder arthroplasty that showed os acromiale type B. (A) Preoperative axillary view X-ray showed a type B os acromion finding. (B) T2-weighted coronal plane image showed a type B os acromion finding. (C) Anteroposterior postoperative X-ray showed no significant difference in the acromion before and after surgery. (D) One year later, the acromiale showed no morphological changes on anteroposterior postoperative X-ray. (E) One year later, the acromiale showed no morphological changes on postoperative computed tomography. Undefined (arrows, meso-acromion). 
satisfied with the surgery with a VAS score of 1 . The ROM, pain, and patient satisfaction were comparable to those of patients who underwent RSA without os acromiale. The UCLA shoulder score also increased significantly from preoperative 12 of 35 (34.3\%) to postoperative 30 of 35 (85.7\%).

\section{DISCUSSION}

In this report, we compared pre- and postoperative parameters in two patients with os acromiale who underwent RSA with cuff tear arthropathy. The results indicated that the procedure improved the ROM, leading to decreased pain and increased patient satisfaction. The incidence of os acromiale varies and has been reported to be around 15\%, with a higher incidence among African Americans (13.2\%-18.2\%) than in Caucasians (5.8\%-9.5\%). However, compared to other ethnic groups, the incidence among Koreans is low at $0.7 \%[6]$.

Os acromiale can be treated conservatively in asymptomatic cases or with surgery in cases with symptoms. Surgery is mainly comprised of excision, acromioplasty, and fixation [2,7]. Though no direct connection with rotator cuff tear has been demonstrated [1], some have argued that the lateral fragment of the os acromiale can be downsloped and cause impingement [8]. Acromial tilt is preferable to acromial downsloping. The conditions of the acromion, clavicle, and scapular spine are important assessments for RSA because they affect tension on the deltoid muscles [9]. Acetabulization can occur in patients with massive rotator cuff tears, thinning, fatigue fracture, or fragmentation of the acromion and is classified according to Hamada grade $[3,10]$.

Os acromiale has been incidentally identified on radiographs. Many cases of os acromiale are asymptomatic, though patients with cuff tear arthropathy can experience acromion weakening due to acetabulization. This is accompanied by os acromiale and affects deltoid function. In these cases, careful attention should be paid to RSA surgery and treatment approaches. We performed RSA using a subscapularis tendon and pectoralis major muscle-sparing approach [5]. No symptoms were associated with os acromiale. The lateral fragment of the os acromiale was slightly disturbed during the operation, but no further treatment, such as removal of fixation, was performed. Other authors have argued that os acromiale itself does not affect surgical outcomes and is not a contraindication to surgery [4,9]. Although radiographs in the present case showed a slight increase in acromial downsloping, the patient did not complain of pain or discomfort at the acromial end.

A few reports have discussed outcomes of RSA in patients with os acromiale, including those by Aibinder et al. [4] in 2017, and
Walch et al. [9] in 2009. However, cases of Asians, especially Koreans, have not been reported. Therefore, due to lack of cases reported for comparisons, the number of cases in the present study was not sufficient to compare or determine statistical significance. Thus, in patients with cuff tear arthropathy with os acromiale, RSA showed a change in postoperative acromial downsloping but did not affect the outcome. The VAS, ROM, and satisfaction of patients improved after compared to before surgery. However, careful attention must be given to surgery and appropriate approach, and follow-up is needed to ensure optimal patient recovery.

\section{ORCID}

Woo-Jin Shin https://orcid.org/0000-0001-5606-8635

Hyun-Ju Lee

Ki-Yong An https://orcid.org/0000-0002-1696-0976 https://orcid.org/0000-0003-3583-6425

\section{REFERENCES}

1. Ji JH, Kim WY, Park SE, Kim YY, Moon CY. Operative treatment of symptomatic Os acromiale. J Korean Shoulder Elbow Soc 2008;11:123-30.

2. Spiegl UJ, Millett PJ, Josten C, Hepp P. Optimal management of symptomatic os acromiale: current perspectives. Orthop Res Rev 2018;10:1-7.

3. Yammine K. The prevalence of Os acromiale: a systematic review and meta-analysis. Clin Anat 2014;27:610-21.

4. Aibinder WR, Schoch BS, Cofield RH, Sperling JW, Sánchez-Sotelo J. Reverse shoulder arthroplasty in patients with Os acromiale. J Shoulder Elbow Surg 2017;26:1598-602.

5. Chung YW, Seo JW, An KY. Subscapular and pectoralis major sparing deltopectoral approach for reverse total shoulder arthroplasty. Clin Shoulder Elbow 2019;22:110-2.

6. Kumar J, Park WH, Kim SH, Lee HI, Yoo JC. The prevalence of os acromiale in Korean patients visiting shoulder clinic. Clin Orthop Surg 2013;5:202-8.

7. Purnell JA, Bourget-Murray J, Kwapisz A, Bois AJ, LeBlanc J. Clinical results and complications following surgical management of symptomatic os acromiale: a systematic review. J Orthop Surg Res 2019;14:26.

8. Hutchinson MR, Veenstra MA. Arthroscopic decompression of shoulder impingement secondary to Os acromiale. Arthroscopy 1993;9:28-32

9. Walch G, Mottier F, Wall B, Boileau P, Molé D, Favard L. Acromial insufficiency in reverse shoulder arthroplasties. J Shoulder Elbow Surg 2009;18:495-502. 
10. Hamada K, Fukuda H, Mikasa M, Kobayashi Y. Roentgenographic findings in massive rotator cuff tears: a long-term observation. Clin Orthop Relat Res 1990;(254):92-6. 